\title{
Formación inicial de profesores de biología en el marco del conocimiento profesional del profesor II -Hallazgos -
}

Biology teachers initial formation in the framework of teacher's professional knowledge II Findings-

Édgar Orlay Valbuena Ussa1, Alice Marcela Gutiérrez', Mónica Correa ${ }^{2}$, Elías Amórtegui ${ }^{2}$

${ }^{1}$ Docentes del Departamento de Biología de la Universidad Pedagógica Nacional. ${ }^{2}$ Estudiantes de décimo semestre de Licenciatura en Biología de la Universidad Pedagógica Nacional. Grupo de Investigación Conocimiento Profesional del Profesor de Ciencias. edgarorlay@hotmail.com ; valbuena@pedagogica.edu.co

\section{Resumen}

Presentamos resultados parciales de la investigación Construcción del Conocimiento Profesional del Profesor de Biología (CPPB), en el Proyecto Cumiallar de Licenciatura en Biología (PCLB) de la Universidad Pedagógica Nacional, particularmente sobre los aspectos formativos de dos actividades curriculares (diseño de Unidades Didácticas -UD- y formulación de anteproyectos pedagógicos APP-).

El desarrollo de estas actividades posibilitó en los futuros profesores: identificar la Biología, la Didáctica y la Pedagogía como componentes del CPPB y la relevancia de éstos en su formación; desarrollar habilidades acerca del Conocimiento Didáctico del Contenido Biológico (CDCB) explicitando su trascendencia en la enseñaza de la Biología; reconocer la importancia de los conocimientos de origen académico y los saberes provenientes de las experiencias y concepciones en su formación; reconocer críticamente los aportes de estas dos estrategias formativas y del PCLB en la construcción de su CPPB.

Destacamos como aportes de la práctica docente integral del PCLB que realizan los futuros docentes en los primeros seis semestres: contribuir a la formación investigativa; aproximar a las realidades educativas; permitir la delimitación de problemas de la educación; posibilitar la continuidad de procesos formativos que orientan las prácticas docentes, la vinculación a líneas de investigación y el desarrollo de trabajos de grado.

\section{Palabras clave}

Formación de docentes, Enseñanza de las Ciencias, Conocimiento Profesional del Profesor de Biología (CPPB), Conocimiento Didáctico del Contenido Biológico (CDCB), Conocimiento Biológico (CB), Proyecto Curricular de Licenciatura en Biología (PCLB).

\section{Abstract}

We present partial results of the research called Constnuction of Biology teacher's professional knowledge in the Biology teacher degree of the Universidad Pedagógica Nacional, emphasizing the identification of educative aspects, through the design of didactic units and pedagogical drafts formulation that contribute to future Biology teachers so that they can construct explicity professional knowledge. 
This activities enabled future teachers to identify Biology, didactics and pedagogy as the main components of Biology teacher's professional knowledge and reflect upon their relevance in their formation, develop skills which match up with the didactic knowledge of biological content asserting its significance for Biology teaching, recognize the importance of knowledge from academic origin and the knowledge whose principal sources are experiences and conceptions in their education as teachers, recognize the contribution of these educative strategies critically and of the Biology degree curricular project in the construction of Biology teacher's professional knowledge.

We stand out the contributions of all around teaching practicum of the PCLB that future teachers carry out in the first six semesters: contribute to the investigative training, approach students to educational contexts, allow Biology education and teaching problems delimitation, and make possible the continuity of educational processes, the connections with research lines and the development of graduation papers.

\section{Keywords}

Teachers training, Sciences teaching, Biology teacher's professional knowledge, Didactic knowledge of the biological content, biological knowledge, Biology degree curricular project.

\section{Introducción}

El presente estudio se circunscribe en los referentes teóricos de la Línea de Investigación del Conocimiento Profesional del Profesor (la cual está relacionada estrechamente con la Línea del Pensamiento del Profesor). Dado que este trabajo constituye la continuación de la ponencia Formación Inicial de Profesores de Biología en el Marco del Conocimiento Profesional del Profesor I. -Contextualización y Fundamentación Teórica -, presentada en este mismo Congreso, no nos detendremos en lo que atañe a la descripción de las características del Proyecto Curricular de Licenciatura en Biología (PCLB), ni en el marco referencial general de la Línea de Investigación recién citada. A respecto, solamente hacemos énfasis en la escasez de investigaciones sobre las investigaciones sobre el Conocimiento Profesional del Profesor de Biología (CPPB). Los aportes que se han hecho, al respecto, hasta el momento son apenas incipientes, los estudios se enfocan fundamentalmente a la relación entre el Conocimiento Biológico que posee el docente y su incidencia en la enseñanza. De la revisión realizada por nuestro Grupo, tan sólo un estudio reporta la progresión de las concepciones de los docentes en formación acerca del conocimiento profesional del profesor de Biología (Valbuena, 2007). Además, en ninguna de dichas investigaciones se aborda el problema de la incidencia de los procesos de formación del profesorado en la construcción de dicho conocimiento.

Así, los estudios realizados con profesores de Biología tanto experimentados como inexpertos y en formación inicial, hacen referencia en especial respecto a los contenidos conceptuales y a la estructura sustantiva del Conocimiento Biológico. No obstante, algunos estudios también hacen referencia a la estructura sintáctica de dicho Conocimiento Disciplinar. En menor proporción, las investigaciones versan sobre el conocimiento pedagógico y sobre el Conocimiento Didáctico del Contenido Biológico (CDCB).

\section{Metodología}

La presente investigación corresponde a un enfoque cualitativo. La principal fuente de información está constituida por los documentos escritos producidos por los futuros profesores durante el desarrollo de dos actividades formativas al interior del PCLB consistentes en: el diseño de Unidades Didácticas (UD) para la enseñanza de temáticas de Biología y la formulación de Anteproyectos Pedagógicos (APP) sobre una problemática de la Educación en Biología. Los 
procesos de dichas actividades se describen en la ponencia complementaria a la presente. Cabe destacar que en la investigación se tuvieron en cuenta más fuentes de información (entrevistas a docentes en formación, entrevistas a formadores, programas de las diferentes asignaturas, guiones para la realización de las actividades de integración, etc.); sin embargo, por limitaciones de espacio no nos referiremos a todos los datos.

Para efectos de esta investigación tuvimos en cuenta el proceso formativo de dos grupos de estudiantes que diseñaron UD para la enseñanza de las temáticas: Cido y división celular $y$ su relación con el crecimiento de los organismos, y Fotoirnitabilidad. En lo que atañe a los datos empleados respecto a la formulación de APP, sistematizamos los correspondientes a auatro propuestas.

Procedimos a identificar en los diferentes documentos producidos por los docentes en formación la información relacionada fundamentalmente con las categońas Conocimiento Didáctico del Contenido Biológico (CDCB) y Conocimiento Biológico (CB), tomando como base el sistema de categorías propuesto por Valbuena (2007). Además, desarrollamos otra categońa correspondiente a los Aspectos Curriculares Formativos (ACF). La sistematización la realizamos utilizando el método de análisis de contenido (Bardín, 1977, y Pérez-Serrano, 1994), para ello inicialmente codificamos las unidades de información, posteriormente ubicamos cada unidad en la correspondiente subcategoría de investigación, enseguida (al interior de las diferentes subcategorías) agrupamos las unidades que compartían referentes y formulamos las respectivas proposiciones.

\section{Resultados y discusión}

Para cada uno de los aspectos de la investigación presentamos la respectiva descripción ilustrando con algunas evidencias (fragmentos de transcripciones de los productos de los futuros profesores, en los cuales destacamos alguna información subrayando las ideas principales) y explicitando las correspondientes implicaciones formativas.

La biología, la didáctica, la pedagogía, la historia, la epistemología, y el conocimiento del contexto como componentes integradores del CPPB.

Los futuros profesores, mayoritariamente hacen énfasis en la integración de los conocimientos pedagógico, didáctico, biológico, histórico-epistemológico de las ciencias. Esto, tanto en la dimensión conceptual (destacando principalmente los modelos pedagógicos), como en la procedimental (habilidades para la formulación de objetivos, la organización de contenidos y actividades de enseñanza).

[Objetivo Formativo al diseñar UD] "Enriquecer algunos conocimientos biológicos, pedagógicos y didácticos que aporten tanto a la planeación y elaboración de la unidad didáctica como a nuestra formación integral profesional." (Estudiantes de un grupo de trabajo de V Semestre del PCLB, al diseñar la UD)

De igual manera la mayoría de futuros docentes hacen énfasis en la importancia del conocimiento político que ha de poseer el profesor, en la medida que le permita tomar posiciones y responder con su función social.

[Justificación de la UD] "El proceso de indagación posibilita tomar conciencia de la importancia y trascendencia de la labor del maestro, como sujeto ético y político y la responsabilidad que tiene en la construcción de sujetos y por tanto de la sociedad a partir de la una disciplina como la biología " (Estudiantes de un grupo de trabajo de V Semestre del PCLB, al diseñar la UD) 
Destacmaos que los docentes en formación no reducen el CPPB a lo biológico. Es así como reconocen que en la formación del profesor de Biología y la planificación de enseñanza que él realice es importante tanto lo disciplinar biológico como lo contextual.

"la formación de Licenciados en Biología requiere de un profundo análisis de las necesidades y las realidades educativas que tiene su localidad, su región y su país: esto implica que el maestro en formación se reconozca dentro de un contexto específico y logre encontrar la función social que debe aumplir". (Estudiante de VI Semestre del PCLB, al formular APP)

En lo que atañe a las fuentes del CPPB, resaltamos que para los estudiantes de quinto semestre predomina la fuente académica, al resaltar como componentes de dicho conocimiento la Biología, la Pedagogía, la Didáctica, la Historia y la Epistemología. En contraste los estudiantes de sexto semestre, durante el proceso de formulación del APP destacan como fuentes tanto la experiencia personal como los conocimientos de origen académico.

"esta construcción es el resultado de mis intereses personales, que se han reforzado durante el transaurso de la carrera, de las relaciones que he establecido entre la academia y mi vida cotidiana... "(Estudiante de VI Semestre del PCLB, al formular APP)

Lo anterior lo podemos explicar por la dinámica propia de las dos estrategias formativas que estamos analizando. Así, para el caso de VI semestre los formadores realizan actividades para explorar en los estudiantes-formadores las experiencias en sus vidas personales y como estudiantes, las cuales hayan podido incidir en la visión que tienen del profesor de Biología.

En las dos actividades los futuros profesores de Biología, reflexionan sobre los saberes y conocimientos con los que han de contar para poder enseñar. Es así como las dos actividades formativas promueven la reflexión en la acción (asumimos que la planificación de la enseñanza también es una actividad práctica).

Las estrategias formativas del PCLB contribuyen al desarrollo de habilidades propias del CDCB.

Las actividades formativas del PCLB a las que hacemos referencia en esta ponencia hicieron posible que los estudiantes-profesores identificaran, desarrollaran y reflexionaran (sobre la importancia en la formación docente) las siguientes habilidades que son necesarias para que el profesor de Biología enseñe de manera más conciente, crítica y regulada las diferentes temáticas biológicas en la escuela:

> Definición de finalidades de la enseñanza tomando como referencia aspectos consustanciales de la Biología y muy especialmente aspectos más cercanos a las características de los alumnos y su entomo (condiciones socioeconómicos, problemas particulares del contexto, intereses, necesidades, etc.). Al respecto, destacamos que para el diseño de la UD los futuros profesores no se limitan a los análisis desde las perspectivas disciplinar biológica y curricular, sino que tienen en cuenta la caracterización de los alumnos y las instituciones escolares, las cuales realizan como parte de la actividad formativa en auarto semestre del PCLB. Así, la mayoría considera como finalidades, la interrelación entre conceptos biológicos estructurantes y de estos con actitudes personales de los alumnos tales como el autocuidado. 
"Otra conducta que se considera pertinente promover es el auto cuidado frente al cáncer, mostrando cómo este se relaciona con procesos de división celular desordenada o anormal, que puede estar directamente influenciado por la exposición a una radiación, entre otros factores, que pueden llegar a generar dicha enfermedad, " (Estudiantes de un grupo de trabajo de V Semestre del PCLB, al diseñar la UD)

$>$ Una de las habilidades que a nuestro parecer se desarrolla más, en relación con el CDCB, es la Estructuración de contenidos de enseñanza a partir de la identificación y reestructuración de conceptos biológicos estructurantes (con el correspondiente establecimiento de relaciones entre estos y otros conceptos), y la consecuente estructuración de contenidos de enseñanza. Para el caso del diseño de la UD, en un primer momento los futuros profesores seleccionan los conceptos que consideran estructurantes, los cuales decantan y explican en redes de conceptos; a partir de la reflexión se percatan que no es suficiente con establecer una estructura de conceptos desde la lógica meramente disciplinar, dado que también cuentan aspectos tales como: las características de los alumnos a quienes está dirigido el material (edades, intereses, concepciones, experiencia), las demandas curriculares y las características del entomo. Así, en momentos posteriores, los estudiantes-profesores realizan una transformación de los conceptos biológicos en contenidos de enseñanza en los que implican algunos conceptos biológicos, procedimientos y actitudes. Respecto este punto, resaltamos que el proceso formativo le posibilita a los futuros profesores hacer conciente que la enseñanza de la Biología demanda transformar e integrar diferentes conocimientos y saberes para llegar a la estructuración de contenidos de enseñanza, los cuales incluyen tanto conceptos como procedimientos y actitudes.

"Los contenidos conceptuales se escogieron como los más importantes para abordar el tema de la unidad didáctica los siguientes: Organismo, creaimiento del organismo, cido celular, mantenimiento. Estos cuatro conceptos se constituyen como los más importantes, porque a partir de ellos los estudiantes pueden llegar a relacionar el crecimiento de los diferentes organismos teniendo una relación con la célula." (Estudiantes de un grupo de trabajo de V Semestre del PCLB, al diseñar la UD)

Para el caso de la formulación de APP, un fuerte componente es la formación ciudadana, contemplando no sólo los contenidos conceptuales, sino también los procedimentales y los actitudinales, siendo el último el más privilegiado, ya que continuamente se resalta lo axiológico como uno de los logros que se deben promover en el aprendizaje de la Biología.

"En Bogotá si hay ranas (la colostethus no es la única) que se están extinguiendo y no solo las ranas sino otras especies también y si no hacemos algo en muy poco tiempo y sin ser exagerada ya no tendremos especies (fauna, flora, etc.) en la Sabana de Bogotá. Lo anterior se debe (creo) a que en la escuela lo único que se enseña de conservación es recidaje, pues estuve buscando artíallos afines y lo único que encontré de ranitas es cómo hacer una disección, cada vez me convenzo más que como futuros licenciados en Biología estamos llamados a innovar, así tal ves cambiarán un poco las cosas..." (Estudiante de VI Semestre del PCLB, al formular APP)

$>$ Durante el desarrollo de las actividades formativas los estudiantes-profesores identifican las dificultades propias de la enseñanza de la Biología, a la vez que plantean altemativas para su superación.

"Para lograr avanzar en estas dificultades (relacionar crecimiento con procesos celulares) se constituye como un elemento importante la historia y la epistemología de las ciencias, pues partiendo de esto se podría lograr que los estudiantes comprendieran la importancia del estudio de la célula, lo cual permitió entender 
muchos de los procesos fisiológicos en los organismos." (Estudiantes de un grupo de trabajo de V Semestre del PCLB, al diseñar la UD).

$>$ Respecto a las actividades de enseñanza, destacamos que las propuestas de los estudiantes-profesores están fundamentadas en referentes de modelos pedagógicos. Es decir, no se trata de un reduccionismo "activista", por el contrario lo planificado se contrasta con los referentes pedagógicos, sustentando cada una de las actividades. Por ejemplo, en concordancia con los modelos de perspectiva constructivista plantean la necesidad de indagar las ideas, generar situaciones problemas, promover la participación de los alumnos y realizar trabajos prácticos en el aula.

"El modelo de aprendizaje por investigación dinigida se identifica por desarrollar el proceso enseñanza - aprendizaje a través de la experimentación, organización, interpretación y análisis de datos, actividades que se involucran en la unidad didáctica por medio del planteamiento de diseños experimentales y de prácticas de laboratorio" (Estudiantes de V Semestre del PCLB)

"Lo que se busca es poder hacer una formulación educativa pertinente para una comunidad afectada por graves condiciones de conflicto social, político, armado, aultural y económico $y$ además por un fuerte impacto ambiental que ha conllevado a la casi desaparición total del humedal." (Estudiante de VI Semestre del PCLB, al formular APP).

$>$ Otro elemento propio del CDCB que presentamos es la relevancia que la asignan los futuros profesores a las ideas de los alumnos.

"La segunda actividad es una encuesta en donde se recogen las ideas previas acerca de los conceptos eje de la UD (sistema viviente, luz, energía, imitabilidad) porque como dice Ausubel y siguiendo el modelo constructivista, el conocimiento y las experiencias previas de los estudiantes son las piezas dave de la conducción de la enseñanza." (Estudiantes de V Semestre del PCLB)

Las particularidades del Conocimiento Escolar (CE)

Las actividades formativas posibilitan que los futuros profesores identifiquen que en la escuela se produce un conocimiento con una epistemología particular. Consideran que la producción del CE no se reduce a una simplificación del conocimiento cient́fico sino que es el resultado de la integración de dicho conocimiento con el cotidiano; destacando que es el conocimiento didáctico el que posibilita dicha integración, mediante la transposición didáctica. Además le confieren al CE un carácter emergente.

[La luz] "...También interviene en aspectos más químicos como la fotosíntesis y la secreción hormonal, lo que da la posibilidad de integrar desde una didáctica de las ciencias el conocimiento cotidiano (lo que el niño ve alrededor) con el conocimiento científico (el por qué y cómo, de lo que el niño ve y no ve con sus ojos) hacia la construcción de un conocimiento escolar más significativo" (Estudiantes de V Semestre del PCLB al diseñar UD)

Elementos del PCLB que potencian la construcción del CPPB.

Reconocemos que las actividades curriculares objeto de esta ponencia favorecen la construcción de un CPPB en los futuros profesores, a partir de un proceso de reflexión en la acción y sobre la acción, que según Schön (1992) se caracteriza como un análisis lógico de eventos y situaciones, sistemáticamente estructurado, deliberado y deliberativo, de los eventos y situaciones, que típicamente es emprendida tiempo después de un evento acontecido. En este caso corresponde al 
reconocimiento de los estudiantes-profesores sobre sus desarrollos profesionales al desarrollar la actividad (diseño de UD, ó formulación de APP), o durante los primeros semestres del PCLB. Según Gutiérrez (2008) "aunque estos profesores están en formación inicial, cada uno de ellos a partir de las diferentes experiencias vividas en el transaurso de la carrera han construido un saber relacionado con su día a día como futuros profesores de Biología...". Al respecto destacamos que en las actividades de integración del PCLB se lleva al docente en formación a explicitar y problematizar sus experiencias en la escuela, sus visiones de la Biología, de la enseñanza, del aprendizaje, de la formación como profesores de Biología. Al respecto estamos de acuerdo con Putman y Broko (1997) que en la formación inicial de docentes es necesario "...atender adecuadamente al conocimiento y creencias que los profesores en formación traen consigo, los auales son adquiridos a lo largo de su experiencia como estudiantes". Aunque no es explíato el interés de los formadores del PCLB desarrollar, en los futuros profesores, habilidades metacognitivas sobre los procesos de formación docente, es decir aquellos que dan cuenta sobre como "aprender a enseñar", consideramos que los estudiantes del PCLB están logrando no sólo la identificación de los componentes que requiere un profesor para poder enseñar profesionalmente la Biología, sino que además reconocen que los procesos que desarrollan al diseñar la UD y al formular el APP contribuyen al aprendizaje de contenidos formativos propios de la profesión docente.

Así, consideremos de suma importancia continuar desarrollando este tipo de actividades al interior del PCLB, las auales aportan a formar profesores más autónomos y críticos. Dicha formación contribuye a la dignificación docente mediante la construcción de un CPPB tal que le permita a los futuros profesores: visualizar finalidades de la enseñanza de la Biología acordes no solamente desde la lógica disciplinar biológica, sino teniendo en cuenta las realidades contextuales; reconocer la importancia de las fuentes experiencial y académica en la estructuración de su conocimiento; identificar la existencia de un Conocimiento Biológico Escolar, el aual se construye mediante el direccionamiento profesional que lo confiere el CDCB del profesor; estructurar objetivos, contenidos y actividades de enseñanza tomando como citerio la transformación e integración didáctica de los distintos saberes y conocimientos que confluyen en la escuela.

\section{Bibliografía}

Bardín, L (1977). Analyse de contenu. Paris: Presses Universitaries de France. (Tra. cast. Análisis del contenido. Madrid: Akal, 1986).

Gutiérrez, A (2008) Uso de estrategias metacognitivas y conocimiento profesional del profesor. Tesis maestría Bogotá: Pontificia Universidad Javeriana.

Pérez y Serrano (1994) investigación cualitativa. Retos e interrogantes (II técnicas y análisis de datos). Madrid: La Muralla, S.A.

Schön, D, (1992). La formación de profesionales reflexivos. Hacia un nuevo diseño de la enser̃anza y el aprendizaje en las profesiones. Barcelona: Paidós.

Valbuena, E. (2007). El conocimiento profesional del profesor de Biología. Estudio de las concepciones de futuros docentes de la Universidad Pedagógica Nacional (Colombia). Tesis doctoral. Universidad Complutense de Madrid. 